\title{
Wave function statistics at the symplectic two-dimensional Anderson transition: Bulk properties
}

\author{
A. Mildenberger ${ }^{1}$ and F. Evers ${ }^{2,3}$ \\ ${ }^{1}$ Fakultät für Physik, Universität Karlsruhe, 76128 Karlsruhe, Germany \\ ${ }^{2}$ Institut für Nanotechnologie, Forschungszentrum Karlsruhe, 76021 Karlsruhe, Germany \\ ${ }^{3}$ Institut für Theorie der Kondensierten Materie, Universität Karlsruhe, 76128 Karlsruhe, Germany
}

(Received 27 October 2006; published 5 January 2007)

\begin{abstract}
The wave function statistics at the Anderson transition in a two-dimensional disordered electron gas with spin-orbit coupling is studied numerically. In addition to highly accurate exponents $\left(\alpha_{0}=2.172 \pm 0.002\right.$, $\tau_{2}=1.642 \pm 0.004$ ), we report three qualitative results. (i) The anomalous dimensions are invariant under $q$ $\rightarrow(1-q)$ which is in agreement with a recent analytical prediction and supports the universality hypothesis. (ii) The multifractal spectrum is not parabolic and therefore differs from behavior suspected, e.g., for (integer) quantum Hall transitions in a fundamental way. (iii) The critical fixed point satisfies conformal invariance.
\end{abstract}

DOI: 10.1103/PhysRevB.75.041303

PACS number(s): 72.15.Rn, 05.45.Df

Disordered electron systems that are confined to two spatial dimensions (2D) cannot support a true metallic state because of Anderson localization. ${ }^{1}$ The underlying physics relates to an interference-enhanced return probability of quantum mechanical particles due to repeated backscattering of the same (quenched) disorder configuration. There are exceptions to the rule, however. For instance, if spin-orbit scattering exists, the return probability is not enhanced but even depleted and the metallic state survives. ${ }^{2}$ Universal properties of such metals are described by the symplectic symmetry class of Gaussian random matrix theories. By increasing the disorder strength $W$, a metal-insulator (i.e., Anderson) transition can be driven in these materials. Its universal properties have been studied intensively in the last two decades.

One of the controversial questions in the late 1990s concerning the symplectic transition in $2 \mathrm{D}$ was about the numerical value of the critical exponent $\nu$ that describes the divergence of the localization length when the disorder approaches its critical value: $\xi \sim\left|W-W_{c}\right|^{-\nu}$. In recent work, Asada et al. have made a very convincing case in favor of $\nu=2.75$ (overview in Table I) employing the $\mathrm{SU}(2)$ model. $^{3}$ A work by Markos and Schweitzer ${ }^{4}$ comes to a similar conclusion, $\nu \approx 2.8 \pm 0.04$, within the Ando model and the debate is now settled.
However, this latter work not only has helped to fix $\nu$, it also has reemphasized that another important topic is still unresolved. Recall that the critical wave functions $\Psi(\mathbf{x})$ at the boundary between insulator and metal obey a multifractal statistics. ${ }^{5}$ This implies that the moments

$$
\left\langle\left\langle|\Psi(\mathbf{x})|^{2 q}\right\rangle\right\rangle \sim L^{-d-\tau_{q}}, \quad q \in \mathbb{R},
$$

scale with system size $L$, introducing the exponent spectrum $\tau_{q}$. (The double angular brackets denote a combined spatial and ensemble average.) A precise numerical determination of $\tau_{q}$ has not been undertaken yet. The numerical work presented in this Rapid Communication is an attempt to close this gap.

There are several good reasons why one would like to scrutinize the nature of $\tau_{q}$ more closely. For one thing, the wave function statistics can be measured, in principle, and promising steps in this direction were made not long ago. ${ }^{6}$

But also important questions concerning our conceptual understanding of the localization-delocalization transition are closely related to multifractality. First, the analytic structure of $\tau_{q}$ is a specific characteristic of the critical field theory of the transition describing scaling of the local density of states. For example, it has been proposed that the (integer) quantum

TABLE I. Overview of results for the symplectic transition in two dimensions. AM: Ando model (Ref. 16); EZM: Evangelou-Ziman model (Ref. 28); MAt (MAa): multifractal analysis based on scaling of typical (average) amplitudes; TM: transfer matrix; SU(2): SU(2) model (Ref. 18); $\delta_{q}$ : reduced anomalous dimension, see Eq. (2). Entries for the same model are in chronological order, starting with the latest work.

\begin{tabular}{|c|c|c|c|c|c|c|c|}
\hline Model & Method & $W_{c}$ & $\Lambda_{c}$ & $\alpha_{0}=2+\delta_{0}$ & $\delta_{q}$ & $\nu$ & Reference \\
\hline $\mathrm{SU}(2)$ & $\mathrm{TM}$ & $5.953 \pm 0.001$ & $1.843 \pm 0.0013$ & & & $2.746 \pm 0.009$ & 3 \\
\hline \multirow[t]{2}{*}{$\mathrm{AM}$} & $\mathrm{TM}$ & $5.838 \pm 0.007$ & $1.87 \pm 0.02$ & & & $2.8 \pm 0.04$ & 4 \\
\hline & MAt & $5.838 \pm 0.007$ & & $2.107 \pm 0.005$ & $\delta_{1}=0.111$ & & 4 \\
\hline $\mathrm{AM}$ & MAt & $5.86 \pm 0.04$ & & & $\delta_{2}=0.19 \pm 0.005$ & $2.41 \pm 0.24$ & 23 \\
\hline $\mathrm{AM}$ & Wave-packet propagation & 5.74 & & & $\delta_{2}=0.15 \pm 0.02$ & & 24 \\
\hline $\mathrm{AM}$ & MAt & 5.74 & & $2.19 \pm 0.03$ & $\delta_{2}=0.17 \pm 0.025$ & & 15 \\
\hline \multirow[t]{2}{*}{ EZM } & MAa & & & & $\delta_{1}=0.16 \pm 0.02$ & & 25 \\
\hline & & & & & $\delta_{2}=0.185 \pm 0.01$ & & 26 \\
\hline Network model & & & $1.83 \pm 0.03$ & & & $2.51 \pm 0.18$ & 27 \\
\hline
\end{tabular}


Hall transition exhibits reduced anomalous dimensions $\delta_{q}$,

$$
\tau_{q}=d(q-1)+\delta_{q} q(1-q)
$$

with a special property: $\delta_{q}$ does not depend on $q$, such that $\tau_{q}$ is parabolic and also invariant under $q \rightarrow 1-q$. Very recently, it has been predicted ${ }^{7}$ - based on exact results for the nonlinear $\sigma$ model and invoking the universality hypothesisthat this last symmetry is a general property of all transitions belonging to the conventional Wigner-Dyson classes. That is,

$$
\delta_{q}=\delta_{1-q}
$$

should hold. A numerical verification beyond the framework of the power law random banded matrix model has not been reported yet. This would be an interesting test of universality, since it does not only rely on comparing quantitative values for some few exponents-which has been the usual procedure-but rather refers to the analytic structure of an exponent spectrum. Note that Eq. (3) does not generally hold outside the conventional symmetry classes. The spin quantum Hall effect is an example for a transition in a nonstandard universality class, where Eq. (3) is manifestly violated. ${ }^{8,9}$

Second, lately it has become clear that near boundaries multifractality differs from the bulk type: flat interfaces support their own "surface" spectrum $\tau_{q}^{s}$; in the presence of corners yet another spectrum is superimposed, etc. ${ }^{10}$

Also, in principle, an edge could break a bulk symmetry and thus would not even share the bulk universality class. In fact, the unraveling of surface multifractality could lead to a paradigmatic shift of our present understanding of critical wave function statistics. Clearly, a prerequisite for all this is a detailed knowledge of bulk properties.

Third, finally, a relation between $\delta_{0}$ and the ratio $\Lambda_{c}$ of width and localization length of quasi-1D strips exists:

$$
\Lambda_{c}=1 / \pi \delta_{0}
$$

which is exact if the critical 2D fixed point is conformally invariant. ${ }^{11}$ It is believed that conformal invariance (CI) is a generic property of localization-delocalization transitions in 2D. For instance, it has been demonstrated to hold at the integer quantum Hall transition. ${ }^{12,13}$ Exceptions are not known so far, but Eq. (4) can be used as a test of CI. In this respect, recent numerical results are alarming. It is reported ${ }^{4}$ that $\delta_{0}=0.107 \pm 0.005$ and $\Lambda_{c}=1.87 \pm 0.02$; thus the product $\pi \Lambda_{c} \delta_{0}=0.629 \pm 0.036$ would signal a strong violation of Eq. (4) and therefore absence of CI. ${ }^{14}$

In this Rapid Communication, we present a numerical high-precision study of $\delta_{q}$ at the 2D symplectic transition. Our particular aim is to answer three qualitative questions. (i) Is $\delta_{q}$ a constant, so $\tau_{q}$ is parabolic? (ii) If not, does it obey the symmetry relation Eq. (3) confirming the universality hypothesis? (iii) Is the fixed point conformally invariant?

Most earlier works analyzed typical moments in small ensembles, where finite-size effects make it difficult to obtain reliable error bars. By contrast, we employ scaling of typical and average moments in very large ensembles with big system sizes. Errors can thus be reduced by almost an order of magnitude. In order to cross-check, we analyze the two most important microscopic models. Results thus obtained agree very well. Specifically, we find that $\delta_{q}$ is not a constant and the symmetry relation (3) is satisfied.

On a quantitative level, we obtain $\delta_{2}=0.180 \pm 0.002$ (both models), $\delta_{0}=0.173 \pm 0.003 \quad$ (Ando model), and $\delta_{0}$ $=0.172 \pm 0.002[\mathrm{SU}(2)$ model $]$. Together with Eq. (4) and the earlier result ${ }^{3} \Lambda_{c}=1.843$ we arrive at $\pi \Lambda_{c} \delta_{0}=0.996 \pm 0.012$. Thus numerical evidence is provided that the symplectic fixed point obeys CI, in agreement with general expectations.

Models. We consider a tight-binding Hamiltonian on a two-dimensional square lattice with nearest neighbor coupling

$$
H=\sum_{i, \sigma} \epsilon_{i} c_{i, \sigma}^{\dagger} c_{i, \sigma}+\sum_{\langle i, j\rangle, \sigma, \sigma^{\prime}} V_{i, \sigma ; j, \sigma^{\prime}} c_{i, \sigma}^{\dagger} c_{j, \sigma^{\prime}}
$$

where $c_{i, \sigma}^{\dagger}\left(c_{i, \sigma}\right)$ denotes a creation (annihilation) operator of an electron with spin $\sigma$ on site $i$.

In the Ando model, ${ }^{16}$ the on-site energies $\epsilon_{i}$ are taken independently from the interval $[-W / 2, W / 2]$ with a homogenous distribution. The hopping matrix $V_{i, \sigma ; j, \sigma^{\prime}}$ reflecting the spin-orbit coupling is chosen as

$$
V_{i, \sigma ; i+k, \sigma^{\prime}}=\left[V_{0} \exp \left(i \theta_{k} \sigma_{k}\right)\right]_{\sigma, \sigma^{\prime}}, \quad k=x, y,
$$

with $\sigma_{x}, \sigma_{y}$ denoting Pauli matrices and the parameters $V_{0}$ $=1$ and $\theta_{k}=\pi / 6$. We have determined the critical disorder strength independently via analysis of the critical level statistis. ${ }^{17}$ Our finding $W_{c}=5.85 \pm 0.025$ agrees well with earlier work. ${ }^{4}$

The second model, the SU(2) model, has been introduced by Asada, Slevin, and Ohtsuki. ${ }^{18}$ In addition to the on-site energies $\epsilon_{i}$, now also the hopping matrix $V_{i, \sigma ; j, \sigma^{\prime}}$ is random. It is taken to be uniformly distributed over the entire group $\mathrm{SU}(2)$ using the group invariant (Haar) measure. ${ }^{18}$

$H$ is implemented on square $(L \times L)$-size lattices with periodic boundary conditions. For our numerical diagonalization of the resulting $2 L^{2} \times 2 L^{2}$ matrices we use an inverse iteration routine coupled with direct sparse solvers in order to obtain the eigenvalues and wave functions with energies closest to zero. ${ }^{19}$ (Cf. Ref. 20.)

Multifractal analysis. Our multifractal analysis proceeds by analyzing the scaling behavior of the average moments of wave function amplitudes, Eq. (1).

In order to analyze the critical behavior we take the disorder value $W_{c}=5.84$ (for states at energy zero being critical) in the Ando model. For the $\mathrm{SU}(2)$ model we employ $W_{c}$ $=5.953$ in order to have a mobility edge at energy $\epsilon=1 .^{3}$ The average (1) has been performed over an ensemble of wave functions that have been calculated in systems of sizes $L$ $=16,24,32,48,64,96,128,192,256$ (the last two values were not used in all cases). For each disorder realization 64 wave functions closest to the critical energy have been taken into account; all together the number of wave functions in the ensemble is typically $4 \times 10^{7}(L=16)$ to $3 \times 10^{5}(L$ $=256$ ).

The exponents $\tau_{q}$ are readily extracted from a power-law fit as suggested by Eq. (1). ${ }^{21}$ In Fig. 1 we plot the reduced dimensions $\delta_{q}$ defined in (2) as obtained for both models. It incorporates our three main results. 


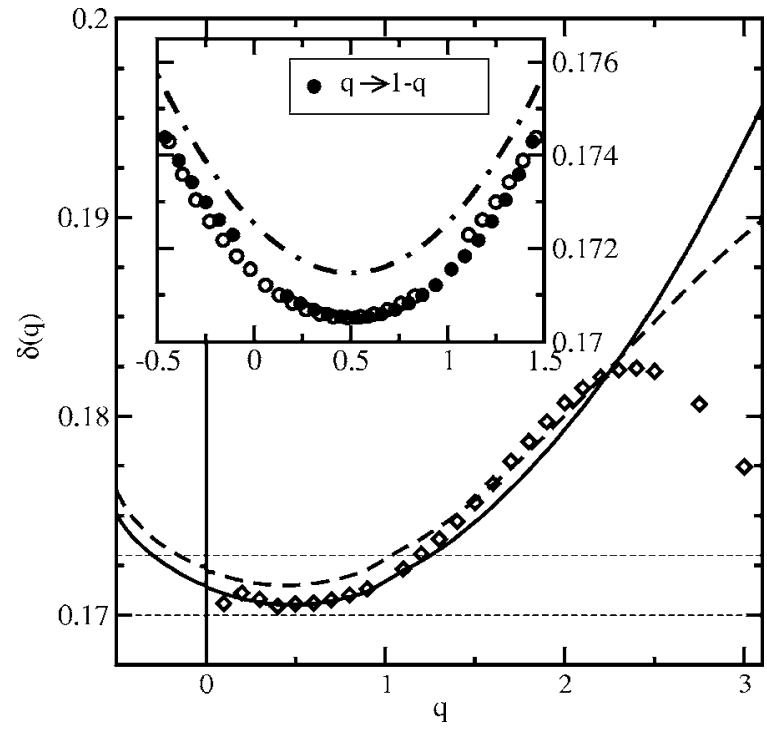

FIG. 1. Reduced anomalous dimension $\delta_{q}$ as defined in Eq. (3) for the Ando model (dashed, $W_{c}=5.84$ ) and the SU(2) model (solid, $W_{c}=5.953$ ). Additionally, anomalous dimensions $\widetilde{\delta}_{q}$ obtained from typical inverse participation ratios are shown $(\diamond)$ for the latter model. Dashed lines indicate the estimated error $(2 \sigma)$ in $\delta_{0}$. Inset: blowup of the solid line behavior near $q=0.5$ now represented by $\bigcirc$. Data near $q=0$ and $q=1$ suffer from noise amplification [dividing by $q(1-q)$ in Eq. (2)] and have therefore been omitted. Filled symbols $(-)$ show original trace after reflection at $q=0.5$. Dotdashed line indicates parabolic fit (offset $10^{-3}$ ) with $\delta_{1 / 2}=0.1705$ and curvature $\delta_{1 / 2}^{\prime \prime}=0.0043$.

(i) We determine $\delta_{0}=0.172 \pm 0.002$. The value satisfies Eq. (4) and thus the consistency check on CI is positive. The good accuracy stems mainly from large statistics and the fact, that finite size corrections in the SU(2) model turn out to be extremely small at $q \lesssim 1.5$. As can also be seen from Fig. 1, the Ando model gives a similar result.

(ii) The function $\delta_{q}$ satisfies the symmetry relation Eq. (3). Thus the universality postulate is confirmed. The inset of Fig. 1 shows that part of the full curve $\delta_{q}$, for which numerical data are available at both points, $q$ and its image $1-q$. [The numerical procedure that we work with is limited to $q$ $\gtrsim-1$; more negative values would require a coarse graining in order to overcome the divergence of the moments (1) related to zeros of the wave functions.] A symmetric shape of the curve is clearly displayed in the regime of best accuracy, $-0.5 \lesssim q \lesssim 1.5$.

(iii) The set of exponents $\delta_{q}$ does not reduce to a constant, e.g., $\delta_{q}$ has a small but nonzero curvature $\delta_{1 / 2}^{\prime \prime}$. Detecting $\delta_{1 / 2}^{\prime \prime}$ requires high-precision data, because the numerical window is limited to $q \lesssim 2.0$. At larger values, (a) finite- $L$ effects proliferate [in the Ando model faster than in SU(2)], so deviations between solid and dashed lines increase. And (b) moments $\left\langle\left\langle|\Psi|^{2 q}\right\rangle\right\rangle$ for large $q$ probe the tails of the distribution function, so that typical values and averages differ from each other. Then, error bars tend to become large due to undersampling. ${ }^{20}$ The parting of the three curves at $q \gtrsim 2$ visible in Fig. 1 is a consequence of these effects.
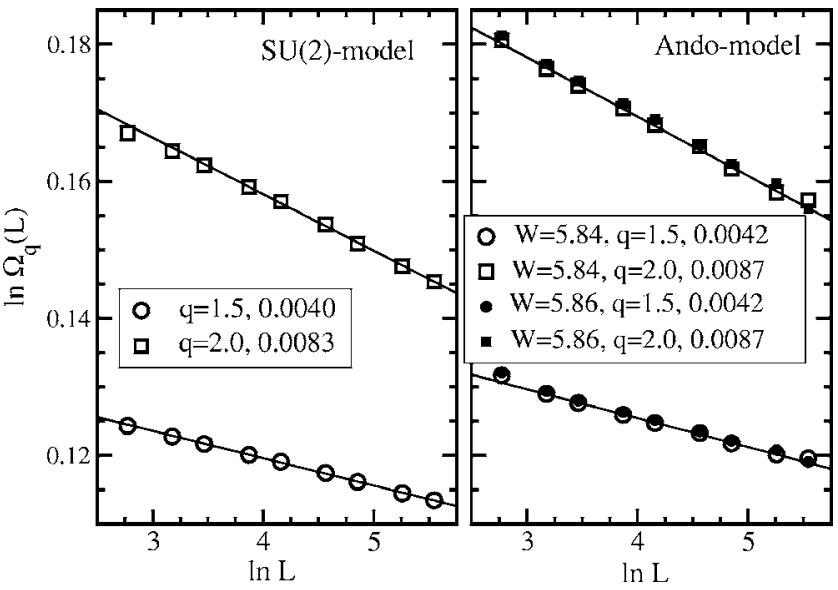

FIG. 2. Test function $\Omega_{q}(L)$ highlighting variability of $\delta_{q}$ with $q$ for two $q$ values. Solid lines represent power law fits, with a slope representing $\delta_{q}-\delta_{1 / 2}$; for values, see legend. Slight deviations between models are due to larger errors in finite-size extrapolation of Ando model. For that model, results for two values of $W$ are given to illustrate that the uncertainty in $W_{c}$ is not a precision-limiting factor. Note that $\delta_{3 / 2}-\delta_{1 / 2}$ agrees well with curvature $\delta_{1 / 2}^{\prime \prime}$ seen in Fig. 1.

As a sensitive test for variability of $\delta_{q}$ we investigate in Fig. 2 the ratio

$$
\Omega_{q}(L)=\left[\left\langle\left\langle|\Psi|^{2 q}\right\rangle\right\rangle L^{d q}\right]^{1 / q(1-q)} /\left[\langle\langle|\Psi|\rangle\rangle L^{d / 2}\right]^{4}
$$

encompassing only unprocessed data. It scales as $\Omega_{q}(L)$ $\sim L^{-\delta_{q}+\delta_{1 / 2}}$ and therefore any slope in $\ln \Omega$ signalizes that $\delta_{q}$ deviates from $\delta_{1 / 2}=0.1705 \pm 0.001$. Data for $\Omega_{q}$ at $q$ $=1.5,2.0$ are shown in Fig. 2 . It clearly exhibits a linear trace with the nonzero slope indicative of curvature in $\delta_{q}$. Note that finite-size effects are very small, so that $\delta_{q}-\delta_{1 / 2}$ can be extracted with good accuracy.

A more conventional object than $\delta_{q}$ to characterize the wave function statistics is the Legendre-transformed $f(\alpha)$ $=q \alpha-\tau_{q}, \alpha_{q}=\partial \tau / \partial q$, displayed in Fig. 3. Even though we

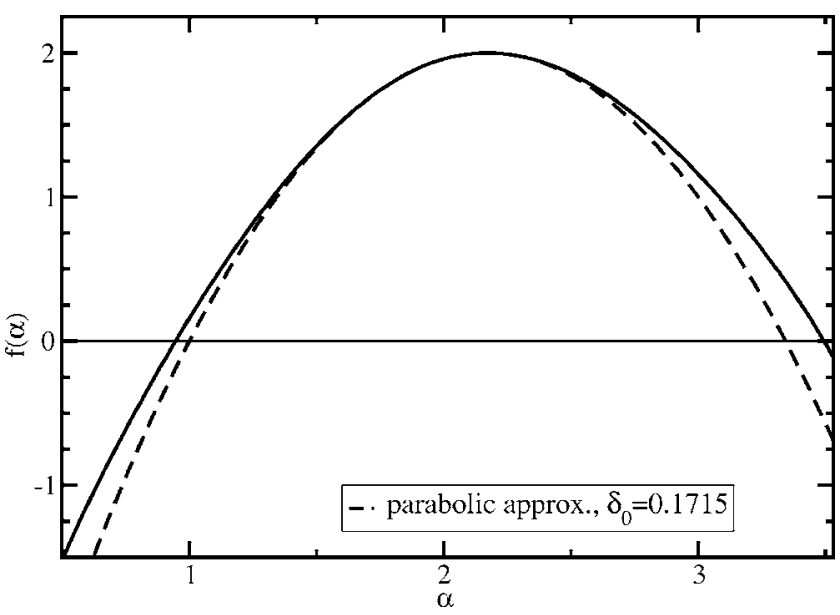

FIG. 3. $f(\alpha)$ spectrum from data of Fig. 1, SU(2) model. $f(\alpha)$ is slightly asymmetric and not a parabolic function, which would have meant $f(\alpha)=2-\left(\alpha-2-\delta_{0}\right)^{2} / 4 \delta_{0}$. 
have obtained $\tau_{q}$ only for $q \gtrsim-1 / 2$ and therefore are restricted to $\alpha \lesssim \alpha_{1 / 2}$, the spectrum can be reconstructed also at values $\alpha \gtrsim \alpha_{1 / 2}$ by making use of Eq. (3). ${ }^{7}$ Then deviations from parabolicity obtrude.

Summary. The multifractal spectrum of wave functions at the 2D symplectic Anderson transition has been calculated in the Ando and SU(2) models with high precision. On a qualitative level, our results demonstrate that the critical fixed point is conformally invariant with a nonparabolic spectrum $\tau_{q}$. Furthermore, $\delta_{q}=\delta_{1-q}$, as predicted from calculations within the nonlinear $\sigma$ model and thus supports the universality hypothesis.

We recently learned about a closely related project, with partly overlapping results. ${ }^{22}$

We thank L. Schweitzer and K. Yakubo for useful correspondence and A. D. Mirlin for valuable discussions and suggestions on the manuscript.
${ }^{1}$ P. Anderson, Phys. Rev. 109, 1492 (1958).

${ }^{2}$ P. Lee and T. V. Ramakrishnan, Rev. Mod. Phys. 57, 287 (1985).

${ }^{3}$ Y. Asada, K. Slevin, and T. Ohtsuki, Phys. Rev. B 70, 035115 (2004).

${ }^{4}$ P. Markos and L. Schweitzer, J. Phys. A 39, 3221 (2006).

${ }^{5}$ A. D. Mirlin, Phys. Rep. 326, 259 (2000).

${ }^{6}$ M. Morgenstern, J. Klijn, C. Meyer, and R. Wiesendanger, Phys. Rev. Lett. 90, 056804 (2003).

${ }^{7}$ A. D. Mirlin, Y. V. Fyodorov, A. Mildenberger, and F. Evers, Phys. Rev. Lett. 97, 046803 (2006).

${ }^{8}$ F. Evers, A. Mildenberger, and A. D. Mirlin, Phys. Rev. B 67, 041303(R) (2003).

${ }^{9}$ A. D. Mirlin, F. Evers, and A. Mildenberger, J. Phys. A 36, 3255 (2003).

${ }^{10}$ A. R. Subramaniam, I. A. Gruzberg, A. W. W. Ludwig, F. Evers, A. Mildenberger, and A. D. Mirlin, Phys. Rev. Lett. 96, 126802 (2006).

${ }^{11}$ M. Janßen, Int. J. Mod. Phys. B 8, 943 (1994).

${ }^{12}$ M. Janßen, M. Metzler, and M. R. Zirnbauer, Phys. Rev. B 59, 15836 (1999).

${ }^{13}$ R. Klesse and M. R. Zirnbauer, Phys. Rev. Lett. 86, 2094 (2001).

${ }^{14}$ The cited value for $\delta_{0}$ is inconsistent with an earlier one, $0.19 \pm 0.03$ (Table I), which would appear to give a much better fit, $1 / \pi \delta_{0}=1.68 \pm 0.3$ (Ref. 15). However, error bars on the earlier estimate are somewhat unclear, because it was obtained with a value $W_{c}=5.74$ that is considerably below more recent findings (Ref. 4), $W_{c}=5.84 \pm 0.007$. In fact, later results by Schweitzer and Zharakeshev for larger system sizes together with accounting for irrelevant scaling terms are compatible with $W_{c}$ from Ref. 4. [L. Schweitzer and I. Zharakeshev (unpublished)].

${ }^{15}$ L. Schweitzer, J. Phys.: Condens. Matter 7, L281 (1995).

${ }^{16}$ T. Ando, Phys. Rev. B 40, 5325 (1989).

${ }^{17}$ We employ a standard procedure [Ref. 15; I. K. Zharekeshev and B. Kramer, Jpn. J. Appl. Phys., Part 1 34, 4361 (1995)] and consider the scaling of the second moment of the normalized level spacing distribution $J(L, W)=(1 / 2) \int_{0}^{\infty} d s s^{2} P_{L, W}(s)$, where $P_{L, W}(s)$ denotes the probability to find an energy difference $s$ between two consecutive eigenvalues. Our renormalization group ansatz incorporating irrelevant scaling fields is $J(L, W)$ $=\widetilde{J}_{R}\left(\phi L^{1 / \nu}\right)+\chi L^{-y} \widetilde{J}_{I}\left(\phi L^{1 / \nu}\right)+\cdots$ with $\phi(W)=\left|W-W_{c}\right| / W_{c}[\chi(W)]$ being the relevant (irrelevant) variables. Results of the scaling analysis are $W_{c}=5.85 \pm 0.025, \nu=2.74 \pm 0.12$, and $y=1.5 \pm 0.5$. The large error of the exponents result from the uncertainty in $W_{c}$.

${ }^{18}$ Y. Asada, K. Slevin, and T. Ohtsuki, Phys. Rev. Lett. 89, 256601 (2002).

${ }^{19}$ R. B. Lehoucq, D. Sorensen, and C. Yang, ARPACK Users Guide (SIAM, Philadelphia, 1998); A. Gupta, M. Joshi, and V. Kumar, IBM Report No. RC 22038 (98932), 2001 (unpublished); P. R. Amestoy, I. S. Duff, and J.-Y. L'Excellent, Comput. Methods Appl. Mech. Eng. 184, 501 (2000); P. R. Amestoy, I. S. Duff, J. Koster, and J.-Y. L'Excellent, SIAM J. Matrix Anal. Appl. 23, 15 (2001).

${ }^{20}$ F. Evers, A. Mildenberger, and A. D. Mirlin, Phys. Rev. B 64, 241303(R) (2001).

${ }^{21}$ In recent papers [H. Obuse and K. Yakubo, Phys. Rev. B 69, 125301 (2004); 71, 035102 (2005)] the width $\sigma(L)$ of the distribution function $\mathcal{P}\left(\tau_{2}\right)$ of $\tau_{2}$ for different system sizes is investigated. A nonzero value in the limit of large system sizes is found, if an approximation scheme is used that assumes power law corrections to scaling, $L^{-|y|}$. However, in reality finite size corrections to $\sigma(L)$ are logarithmic rather than power law. Indeed, the published data [Obuse and Yakubo, Phys. Rev. B 69, 125301 (2004), Fig. 6] are consistent with a slow, logarithmic flow of $\sigma(L)$ to zero in agreement with the self-averaging nature of the multifractal exponents $\tau_{q}$ and in contrast to earlier claims made in the literature [D. A. Parshin and H. R. Schober, Phys. Rev. Lett. 83, 4590 (1999)].

${ }^{22}$ H. Obuse, A. R. Subramaniam, A. Furusaki, I. A. Gruzberg, and A. W. W. Ludwig, cond-mat/0609161 (unpublished).

${ }^{23}$ K. Yakubo and M. Ono, Phys. Rev. B 58, 9767 (1998).

${ }^{24}$ T. Kawarabayashi and T. Ohtsuki, Phys. Rev. B 53, 6975 (1996).

${ }^{25}$ S. N. Evangelou, Physica A 167, 199 (1990).

${ }^{26}$ J. T. Chalker, G. J. Daniell, S. N. Evangelou, and I. H. Nahm, J. Phys.: Condens. Matter 5, 485 (1993) [index in Table I should be shifted: $q \rightarrow q+1$; J. Chalker (private communication)].

${ }^{27}$ R. Merkt, M. Janssen, and B. Huckestein, Phys. Rev. B 58, 4394 (1998).

${ }^{28}$ S. N. Evangelou and T. Ziman, J. Phys. C 20, L235 (1987). 